\title{
Maternal experiences with and sources of information on galactagogues to support lactation: a cross-sectional study
}

This article was published in the following Dove Press journal:

International Journal of Women's Health

27 February 2017

Number of times this article has been viewed

\author{
Alessandra N Bazzano' \\ Lauren Cenac' \\ Amelia J Brandt' \\ Josephine Barnett ${ }^{2}$ \\ Shelley Thibeau ${ }^{3}$ \\ Katherine P Theall' \\ 'Department of Global Community \\ Health and Behavioral Sciences, Tulane \\ University School of Public Health \\ and Tropical Medicine, New Orleans, \\ LA, ${ }^{2}$ City University of New York, \\ CUNY Graduate Center, New York, \\ NY, ${ }^{3}$ Ochsner Clinic Foundation, New \\ Orleans, LA, USA
}

Correspondence: Alessandra N Bazzano Tulane University School of Public Health and Tropical Medicine, 1440 Canal Street, New Orleans, LA 70II2, USA

Tel +l 5049882338

Email abazzano@tulane.edu

\begin{abstract}
An increase in the marketing and use of herbal galactagogues among breastfeeding mothers in the US has raised the issue of how best to provide support and information on the use of these products, particularly in light of limited availability of certified lactation counselors and continued suboptimal rates of breastfeeding globally. Currently, no cross-sectional data are available on the experiences and attitudes of mothers regarding the use of herbal and pharmaceutical galactagogues for lactation in the US. The findings of an online survey of 188 breastfeeding mothers on experiences with and sources of information on galactagogues are presented. Most mothers (76\%) reported that while breastfeeding, they felt as though they were not making enough milk to meet the needs of their child, and yet $54 \%$ also indicated that they had not supplemented with formula. A large proportion of respondents reported utilizing galactagogues to increase lactation and finding them useful. The results indicated that most women learned about galactagogues from the Internet or by word of mouth through friends. Lactation consultants were the third-most reported sources of information on these products. While many respondents reported perceiving galactagogues as innocuous, more evidence on safety and efficacy is needed to support women properly who seek out and use them. Large-scale studies of the prevalence of galactagogue use in the US and rigorous evaluation of use globally are needed to ensure that mothers who choose to breastfeed may safely avail themselves of all options when counseling support is insufficient.
\end{abstract}

Keywords: galactagogue, breastfeeding, lactation, lactation support

\section{Introduction}

Further advances in improving the research and policy environment surrounding breastfeeding in the US are urgently needed to support women in achieving more successful lactation outcomes. Though the maternal and child-health benefits of breastfeeding are well documented, ${ }^{1-6}$ rates of breastfeeding in the US remain suboptimal. ${ }^{7,8}$ Only $18.8 \%$ of infants are breastfed exclusively at age 6 months, and only $26.7 \%$ are still being breastfed at age of 12 months. ${ }^{9}$ Provision of breastfeeding counseling and support is a widely accepted strategy, but women may not be able to access certified lactation consultants, and must seek support from other sources..$^{10,11}$

Current breastfeeding-promotion interventions have not been adequately successful in addressing insufficient milk supply, ${ }^{12-16}$ the most common reason that women cite when they discontinue breastfeeding. ${ }^{15,17-19}$ In a 2001 study, 30\% of mothers reported this as the major reason for discontinuation, ${ }^{15}$ while another study of participants in the Special Supplemental Nutrition Program for Women, Infants and Children (WIC) in the US reported that $46 \%$ who stopped breastfeeding before the recommended 
age did so because of perceived inadequate milk supply. ${ }^{20}$ Consequently, mothers (notably, adoptive mothers seeking to induce lactation) and health care workers involved in supporting them have sought other modes of lactation support to address this concern, including using galactagogues: foods, medicines, herbal supplements, and teas purported to have lactogenic properties. ${ }^{21-27}$ Breastfeeding women have accessed herbal medicines for the purpose of aiding lactation historically and in diverse settings. ${ }^{24,26-41}$ Fenugreek is one substance that has been widely used for lactogenic effect, and as a result has been growing in popularity globally. ${ }^{42}$ The scientific literature documenting the efficacy of herbal galactagogues, such as fenugreek, fennel, blessed thistle, and goat's rue, is quite limited. ${ }^{21,22,24,26,28,32,37,39-41,43-49}$ Galactagogues are assumed to enhance milk supply and production by increasing the level of the hormone prolactin, but ultimately the biological mechanisms by which they may or may not function are not well understood..$^{48}$

Though numerous intervention studies have aimed at supporting breastfeeding continuation and exclusivity through lactation-consultant support, ${ }^{50-59}$ fewer have examined the use of pharmacological or herbal supplements as an additional lactation aid. ${ }^{24,26,32,39,40,60-63}$ Various challenges can impede successful breastfeeding initiation, exclusive breastfeeding during the first 6 months, and continuation. Socioeconomic factors and health disparities result in greater barriers for vulnerable populations, often related to the need to return to work soon after giving birth. ${ }^{64}$ Maternal health factors, including delayed childbearing, cesarean section, multiple births, mode of conception, ${ }^{29}$ and obesity may also contribute to potential challenges to breastfeeding. ${ }^{65}$ Maternal obesity in particular places a mother at increased risk of physiological difficulties during lactation and lower breast-milk production, ${ }^{66}$ and may be associated with insufficient secretion of the hormone prolactin, required for milk production. ${ }^{67}$ Mammary hypoplasia, use of analgesics during labor, and family history of alcohol dependence may also put certain women at risk for challenges with lactation. ${ }^{67}$ In first-time mothers, delayed onset of lactogenesis II can be common, and has been associated with risk of early discontinuation of breastfeeding. ${ }^{31}$ Currently, breastfeeding mothers in the US have very limited access to pharmacological interventions for increasing milk supply, and physicians may only prescribe these "off-label" or not for their intended use; the US Food and Drug Administration has not approved any pharmaceutical products for lactation. ${ }^{27}$ In contrast, Canadian women frequently utilize prescription medication for lactation support. ${ }^{68}$

Given that many herbal and pharmaceutical options are increasingly sought and used, ${ }^{69}$ despite the lack of evidence about their efficacy to increase milk supply, ${ }^{69,70}$ additional studies for improved health information regarding galactagogue use are warranted. Studies involving mothers who have used pharmaceutical and herbal galactagogues are also critical, since participants can provide first-hand insight into experiences with and effects of galactagogue use, as well as sources of information that could be targeted for improvement. ${ }^{33,38}$ Breastfeeding knowledge is a key factor influencing infant feeding, ${ }^{71,72}$ and studies that examine mothers' experience and source of information are valuable in informing clinical guidelines for recommendation, as well as the selection of specific interventions to be tested through controlled trials. ${ }^{30}$ Previous research on this topic has been limited to specific geographic regions, and to date no studies have been conducted in the US through use of a survey administered online. The current cross-sectional study aimed to add to the scarce literature on galactagogues and expand upon the recent studies reporting women's experiences using herbal supplements to improve lactation. ${ }^{24,25,30,46}$

\section{Materials and methods}

The present survey was designed to explore mothers' experiences with galactagogues to improve lactation and sources of information on these products. The online questionnaire format administered in this study was developed to elicit information efficiently on experiences of mothers who were interested in using, currently using, or who had used galactagogues for breastfeeding. An exploratory cross-sectional approach was employed, and the protocol and study materials were reviewed by the Tulane University and Ochsner Clinic Foundation institutional review boards and deemed exempt from further oversight on the basis of research involving survey or interview procedures with adult subjects, as per criteria of 45 CFR 46.101(b)(2) (US Department of Health and Human Services). The STROBE (Strengthening the Reporting of Observational Studies in Epidemiology) checklist for reporting on cross-sectional studies has been used to develop the following sections concerning details of this research, including information on the survey content.

\section{Sampling}

A convenience sample of women who use Internet-based breastfeeding websites was employed. Probability sampling was not possible, due to constraints in obtaining formal access to a list of populations, due to privacy considerations and the lack of a full description of the sampling frame. As a large proportion of women use the Internet for perinatal advice, including on breastfeeding, and numerous websites specific to lactation exist, ${ }^{73}$ the Internet was the primary setting for 
recruitment. In order to participate, mothers had to be aged 18 years, speak English, and have access to the Internet.

\section{Survey}

Participants were enrolled if they chose to click on a survey link, replied affirmatively to consent to participate, and confirmed being 18 years or older in the online survey questionnaire. An invitation to participate was distributed through the Internet, including through social media. Sites on which the survey invitation appeared included the Baby Center, Pinterest, Kelly Mom, and La Leche League websites and various Facebook pages in the New Orleans and Baton Rouge areas. Participants were also encouraged to distribute the survey widely by email or text message to other mothers, for snowball sampling. Doulas and lactation consultants located near the authors' research institutions were asked to send the survey to their contacts.

Data collection began in October 2015 and ended in December 2015. Participants were able to choose the setting in which the online survey was completed: any location with a computer, smartphone, or tablet computer. Individuals were informed that their participation was voluntary, consent was implied upon completion of the survey, and that responses would be anonymous. No incentive was provided, and there was no cost for participants in the survey. The survey included 28 items.

Closed-ended questions varied in format. Some closedended questions had binomial answers of either yes or no. Other questions allowed respondents to choose only one response, but these responses were not binomial. For example, the question "How many children have you ever breastfed?" allowed survey respondents to select only one response from several options. Still other closed-ended questions allowed respondents to select multiple answers by checking all that applied. Finally, some questions allowed respondents to choose only one response using a 5-point Likert scale format. Open-ended questions were included directly following closed-ended questions, giving respondents an opportunity to supplement their response with more information. A total of 188 participants took the survey; it was not mandatory that respondents answer every question.

\section{Data analysis}

The survey data were delivered and managed through the Health Insurance Portability and Accountability Actcompliant Qualtrics platform, which uses Transport Layer Security encryption, and then entered into Statistical Package for the Social Sciences (SPSS) for Windows version 23 (IBM Corporation, Armonk, NY, USA) for further analysis.

\section{Results \\ Demographic results}

A total of 188 women from 27 states completed the questionnaire. The majority of respondents were residents of two states: Louisiana (52\%) and Kansas (12\%). Table 1 illustrates the sociodemographic characteristics of the study sample.

The vast majority of respondents were white (92\%) and non-Hispanic (94\%). Most were married (87\%) and

Table I Characteristics of study participants

\begin{tabular}{|c|c|c|}
\hline Characteristic & $\mathbf{n}$ & Percentage \\
\hline \multicolumn{3}{|l|}{ Race } \\
\hline American Indian/Alaska Native & I & 0.6 \\
\hline Asian & 3 & 1.9 \\
\hline Black/African-American & 2 & 1.3 \\
\hline Native Hawaiian/Pacific Islander & 3 & 1.9 \\
\hline White & 144 & 91.7 \\
\hline Other & 4 & 2.6 \\
\hline Total & 157 & \\
\hline \multicolumn{3}{|l|}{ Ethnicity } \\
\hline Hispanic or Latino & 9 & 5.7 \\
\hline Non-Hispanic or Latino & 148 & 94.3 \\
\hline Total & 157 & \\
\hline \multicolumn{3}{|l|}{ Relationship status } \\
\hline Single & 7 & 4.5 \\
\hline Married & 137 & 87.3 \\
\hline With partner & 8 & 5.1 \\
\hline Divorced/separated & 5 & 3.2 \\
\hline Total & 157 & \\
\hline \multicolumn{3}{|l|}{ Number of people residing in home } \\
\hline $\mathrm{I}-2$ & 7 & 4.4 \\
\hline $3-4$ & $12 \mid$ & 77 \\
\hline 5 or more & 29 & 18.5 \\
\hline Total & 157 & \\
\hline \multicolumn{3}{|l|}{ Education } \\
\hline High school or equivalent & 5 & 3.2 \\
\hline Trade school & 9 & 5.7 \\
\hline Some college & 23 & 14.6 \\
\hline Undergraduate degree & 65 & 41.1 \\
\hline Graduate degree & 47 & 29.7 \\
\hline Professional degree & 9 & 5.7 \\
\hline Total & 158 & \\
\hline \multicolumn{3}{|l|}{ Employment } \\
\hline Employed for wages & 90 & 57 \\
\hline Self-employed & 19 & 12 \\
\hline Out of work and looking for work & 1 & 0.6 \\
\hline Out of work and not looking for work & 6 & 3.8 \\
\hline Homemaker & 39 & 24.7 \\
\hline Student & 3 & 1.9 \\
\hline Total & 158 & \\
\hline \multicolumn{3}{|l|}{ Income (US\$) } \\
\hline$<10 \mathrm{k}$ & 1 & 0.6 \\
\hline $10 \mathrm{k}-49.9 \mathrm{k}$ & 40 & 25.5 \\
\hline $50 \mathrm{k}-99.9 \mathrm{k}$ & 54 & 34.4 \\
\hline $100 k-150 k$ & 37 & 23.6 \\
\hline$>150 k$ & 25 & 15.9 \\
\hline Total & 157 & \\
\hline
\end{tabular}


lived in a home with three or four people (77\%). Most of the respondents were college-educated, with either an undergraduate $(41 \%)$, graduate $(30 \%)$, or professional degree $(6 \%)$. Among the respondents who noted that they were employed, the majority worked for wages $(57 \%)$ or were self-employed (12\%). Household income levels varied for women who completed the survey. The largest proportion (34.4\%) had a total household income between US\$50,000 and \$99,000, while many also reported a household income between $\$ 10,000$ and $\$ 49,000$ (26\%), or between $\$ 100,000$ and $\$ 150,000$ (24\%). The majority of respondents had given birth to one $(51 \%)$ or two children $(34 \%)$, and had breastfed one $(53 \%)$ or two children $(33 \%)$.

\section{Breastfeeding characteristics and pattern}

The vast majority of respondents $(76 \%)$ reported that while breastfeeding, they felt as though they were not making enough milk to meet the needs of their child. Table 2 includes breastfeeding characteristics and patterns of mothers who responded to the survey. A majority of respondents (54\%) also indicated that they had not supplemented with formula as a result of not making enough milk to meet the needs of their infant. More than half (60\%) of mothers who responded mentioned they had consulted a health care provider about not making enough milk.

\section{Sources of information and support}

With regard to sources of information or support about not making enough milk, the vast majority of respondents $(85 \%)$ indicated that they sought breastfeeding support from sources other than a lactation counselor, physician, or other health care worker. The most common way for mothers to learn about herbal galactagogues was the Internet. Internet sources consisted primarily of such websites as Kelly Mom or Baby Center and Facebook pages, while "other" non-Internet sources noted were books and in-person support groups through organizations like La Leche League and the Special Supplemental Nutrition Program for Women, Infants and Children (WIC). Other reported sources of information included lactation consultants $(54 \%)$, friends $(34 \%)$, family members $(21 \%)$, pediatricians $(20 \%)$, and obstetricians $(16 \%)$.

\section{Use of galactagogues and experiences with these}

Most of the respondents (94\%) did not take a prescription medication to help them produce milk. Among those who noted they took medication $(6 \%)$, obstetricians were most likely (78\%) to prescribe the medication. Most women (59\%)
Table 2 Breastfeeding characteristics of respondents

\begin{tabular}{|c|c|c|}
\hline Characteristic & $\mathbf{n}$ & Percentage \\
\hline \multicolumn{3}{|c|}{ Number of times given birth } \\
\hline 0 (adoptive parent) & I & 0.6 \\
\hline I & 80 & 50.6 \\
\hline 2 & 54 & 34.2 \\
\hline 3 & 17 & 10.8 \\
\hline 4 or more & 6 & 3.8 \\
\hline Total & 158 & \\
\hline \multicolumn{3}{|c|}{ Number of children breastfed } \\
\hline 1 & 94 & 52.8 \\
\hline 2 & 59 & 33.1 \\
\hline 3 & 18 & 10.1 \\
\hline 4 or more & 7 & 4 \\
\hline Total & 178 & \\
\hline \multicolumn{3}{|c|}{ Felt she was not making enough milk } \\
\hline Yes & 134 & 76.1 \\
\hline No & 42 & 23.9 \\
\hline Total & 176 & \\
\hline \multicolumn{3}{|c|}{ Supplemented with formula } \\
\hline Yes & 62 & 46.3 \\
\hline No & 72 & 53.7 \\
\hline Total & 134 & \\
\hline \multicolumn{3}{|c|}{ Consulted a health care provider } \\
\hline Yes & 81 & 60.4 \\
\hline No & 53 & 39.6 \\
\hline Total & 134 & \\
\hline \multicolumn{3}{|c|}{ Sources of support (check all that apply) } \\
\hline Lactation consultant & 101 & 53.7 \\
\hline Friend & 63 & 33.5 \\
\hline Family & 40 & 21.3 \\
\hline Pediatrician & 37 & 19.7 \\
\hline Obstetrician/gynecologist & 30 & 16 \\
\hline Family practice pediatrician & 12 & 6.4 \\
\hline Midwife & 9 & 4.8 \\
\hline Doula & 7 & 3.7 \\
\hline Other & 2 & 1.1 \\
\hline Total & 188 & \\
\hline \multicolumn{3}{|c|}{ Sought information from other sources } \\
\hline Yes & 114 & 85.1 \\
\hline No & 20 & 14.9 \\
\hline Total & 134 & \\
\hline \multicolumn{3}{|c|}{ Took pharmaceutical galactagogue } \\
\hline Yes & 9 & 6 \\
\hline No & $|4|$ & 94 \\
\hline Total & 150 & \\
\hline \multicolumn{3}{|c|}{ Who prescribed pharmaceutical galactagogue? } \\
\hline Obstetrician/gynecologist & 7 & 77.8 \\
\hline Other & 2 & 2.2 \\
\hline Total & 9 & \\
\hline \multicolumn{3}{|c|}{ Provider's recommendation was important } \\
\hline Yes & 23 & 58.9 \\
\hline No & 16 & 41 \\
\hline Total & 39 & \\
\hline
\end{tabular}

who reported taking medication mentioned their health provider's recommendation of the product was important to them. Tables 3 and 4 describe the use of milk-production aids, including herbal and pharmaceutical galactagogues, among the study sample. 
Table 3 Use of milk-production aids

\begin{tabular}{|c|c|c|c|}
\hline Aid & $\begin{array}{l}\text { Heard of } \\
\text { this, } n(\%)\end{array}$ & $\begin{array}{l}\text { Used, } \\
\text { n (\%) }\end{array}$ & $\begin{array}{l}\text { Information source, } \\
\text { n (\% of sample) }\end{array}$ \\
\hline Breast pumping & $160(85.1)$ & $121(64.4)$ & LC: 86 (45.7) \\
\hline Fenugreek & $162(86.2)$ & $86(45.7)$ & Internet: 9I (48.4) \\
\hline Fennel & $95(50.5)$ & $30(16)$ & Internet: 49 (26.I) \\
\hline Milk thistle & $109(58)$ & $24(12.8)$ & Internet: 59 (3I.4) \\
\hline Goat's rue & 37 (19.7) & $4(2.1)$ & Internet: $19(10.1)$ \\
\hline More milk (plus) & 75 (39.9) & $28(14.9)$ & Friend: $19(10.1)$ \\
\hline Metoclopramide & $18(9.6)$ & $3(1.6)$ & Internet: 7 (3.7) \\
\hline Domperidone & $67(35.6)$ & $5(2.7)$ & Internet: 36 (19.1) \\
\hline
\end{tabular}

Abbreviation: LC, lactation consultant.

Among the 188 women who responded to the survey, the majority had heard of $(85 \%)$ and used (64\%) breast pumping or manual expression to increase their milk supply. Women who had heard of breast pumping or manual expression were most likely to hear about this milk-production aid from lactation consultants $(46 \%)$. The majority of respondents also reported they felt breast pumping or manual expression was very safe (98\%), increased their milk supply (70\%), they would use it again (99\%), and would recommend it to a friend (98\%). Fifteen percent of respondents reported experiencing side effects from breast pumping or manual expression, such as breast soreness or chafing.

Among both herbal and pharmaceutical galactagogues, fenugreek was the most well known among respondents $(86 \%)$ and most used (46\%) by those in the study sample. Nearly half (48\%) of the women in the study reported hearing about fenugreek from the Internet. The least-known milkproduction aid among the study sample was the pharmaceutical drug metoclopramide (10\%). The most commonly reported way for mothers to hear about pharmaceutical galactagogues was the Internet. Among all the milk-production aids included in the questionnaire, mothers reported using the two pharmaceuticals metoclopramide (2\%) and domperidone (3\%) the least.

More than half of the women in the sample also reported having heard of other herbal galactagogues, such as fennel (51\%) and milk thistle (58\%), but only $16 \%$ (fennel) and $13 \%$ (milk thistle) mentioned they had used them. A majority of respondents also reported fenugreek (54\%), goat's rue (75\%), and More Milk (56\%) increased their milk supply, whereas most respondents noted they were not sure if fennel (59\%) or milk thistle (52\%) increased their milk supply.

The most commonly cited reason for not using a herbal galactagogue again or recommending one to a friend was that it was not working. Additionally, using an open-ended option for "other" galactagogues, some mothers reported using certain beverages and foods, such as beer, oatmeal, and brewer's yeast, for lactation.

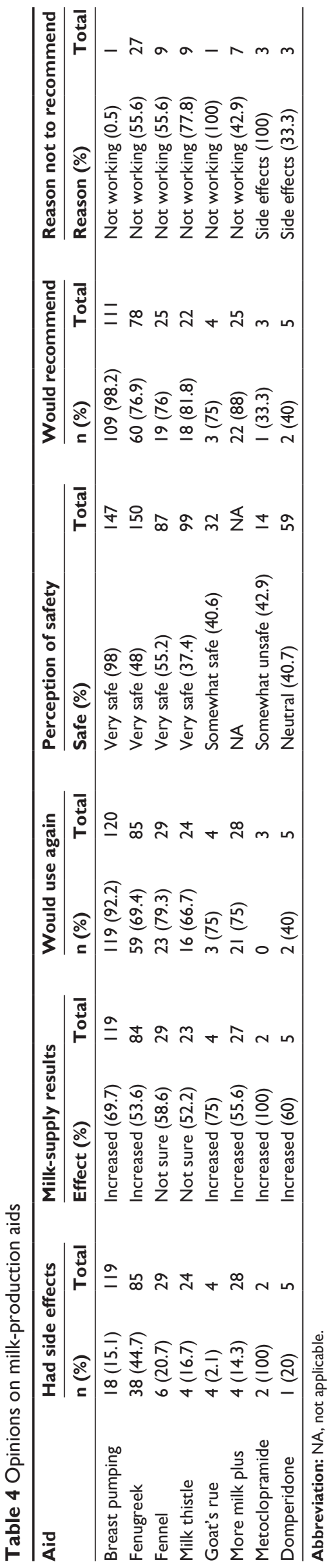




\section{Perception of safety and side effects}

When asked about the safety of herbal galactagogues, herbs that were considered "very safe" included fenugreek (48\%), fennel (55\%), and milk thistle (37\%). Overall, a minority of respondents reported side effects for the herbal galactagogues, and the majority responded they would use them again or recommend them to a friend.

The largest number of respondents reported side effects for fenugreek (45\%) among the herbal galactagogues. Some of the side effects reported by mothers included a maplesyrup smell emitting from the mother's body, gassiness in the baby, or breast-milk oversupply. Among two participants who reported using metoclopramide, both $(100 \%)$ reported side effects. Overall, respondents reported that the pharmaceutical galactagogues increased their milk supply, but they did not consider them to be safe, would not use them again, and/or would not recommend them to a friend. The most commonly cited reason not to recommend a pharmaceutical galactagogue to a friend was side effects. Some of the side effects mothers indicated for pharmaceutical galactagogues were depression (metoclopramide and domperidone) and anxiety (metoclopramide).

\section{Additional analyses}

There were no statistically significant differences in the characteristics of study participants, nor associations with the use of galactagogues or mothers' opinions on herbal supplements. Only two relationships were significant (perception of insufficient lactation based on parity, and consulting a provider based on income level), so logistical models were built for those two relationships. The models prepared were an improvement over the null, but did not have practical significance to the research questions.

\section{Discussion}

Insufficient milk supply is cited as a primary reason for early termination of breastfeeding, and is a very important concern for lactating women, with $30 \%-80 \%$ of breastfeeding mothers globally naming this as a major reason for breastfeeding cessation. ${ }^{74} \mathrm{~A}$ notable finding of the present study was that $76 \%$ of participants felt as though they were not making enough milk to meet the needs of their child, and yet a majority (54\%) chose not to supplement or replace breastfeeding with use of infant formula. This indicates that an important proportion of women have the motivation to continue to find solutions to difficulties that they encounter in lactation and actively seek out support. Up to $15 \%$ of childbearing women in the US may use herbal galactagogues, yet there is scant evidence on safety and efficacy. ${ }^{39}$ Clinicians may also be asked by patients experiencing difficulties in lactation to prescribe drugs "off-label", as in the case of metoclopramide, or through compounding pharmacies, as in the case of domperidone.

Among the very few previous studies that have aimed to explore mothers' perspectives and experiences of using pharmaceutical and herbal galactagogues, ${ }^{24,75,76}$ no study to date has included a sample of US women. This online survey addressed this gap by sampling US-based breastfeeding women to document experiences with, safety of, and usefulness of herbal and pharmaceutical galactagogues, as well as sources of information on these. The current study contributes to efforts aimed at better understanding supportive aids for breastfeeding women to improve success in lactation.

The majority of our study respondents resided in southern areas of the US, a region where breastfeeding rates are $20 \%-30 \%$ lower than the national average, both for initiation and duration of breastfeeding. ${ }^{64,66}$ Given women and children have high rates of preventable chronic diseases, such as obesity, in this region, ${ }^{77}$ breastfeeding has strong potential to improve health outcomes.

Mothers who have reported use of galactagogues, both herbal and pharmaceutical, in an effort to increase milk production for breastfeeding typically report an overall increase in milk supply. ${ }^{23,32,44,45,78,79}$ Our study findings were consistent with these previous studies: nearly all participants who used galactagogues in the current study reported an increase in milk supply.

The present sample consisted primarily of Caucasian women, similar to the composition of a sample from a previous study conducted in Australia. The majority of the Australian study participants, who were not recruited through the Internet, found galactagogues to be effective in increasing their breast-milk supply, and information on galactagogues was based on the recommendations of friends or family members or information they obtained through their own Internet research. ${ }^{24}$ This is in concordance with the present study findings, where the most likely reported source of information was the Internet. Nearly half (48\%) of the women cited the Internet as the main source of information concerning fenugreek, which was also reported to be the most well-known $(86 \%)$ and most used herbal supplement (46\%). Fenugreek was the most effective in perceived, reported increase in milk supply to just over half of the women using it (54\%), but it was also the most reported to have side effects (45\%). However, the overall occurrence of side effects did not influence the report of efficacy or recommendations. 
A large percentage of participants responded that they would use botanical galactagogues in the future. The participants perceived herbals to be safe and effective and would recommend them to others. Pharmaceutical galactagogues were reported as much less likely to be used again, due to side effects. Breast pumping was the second-most familiar milk-production aid used by women, followed by milk thistle, fennel, More Milk, domperidone, goat's rue, and metoclopramide. Almost every method (except fennel and milk thistle) was reported to be working, based on milk supply; however, the recommendations were not as positive. This could be due to the small sample size for "reasons not to recommend". This study revealed that women perceive herbal galactagogues, specifically fenugreek, as most effective in enhancing breast-milk production. The increased use of fenugreek over pharmaceutical galactagogues may be associated with innate comfort, traditional beliefs, high efficacy, and unknown toxicity of medicines. ${ }^{24,30}$ Herbal medicines, however, are less subject to rigorous evaluations for safety and quality compared to registered pharmaceutical medicines. ${ }^{24}$ In contrast, domperidone, with known efficacy and side effects, was less known and used in this study. Domperidone appears to be a safer alternative, and has been shown to be effective in increasing breast-milk volume with a low concentration of medication in breast milk in various small trials. ${ }^{41,60,62,80-83}$ However, pharmaceutical galactagogues, whether due to constraints on their use or safety concerns, are less widely used by the US lactation population. ${ }^{84}$

\section{Limitations}

Study limitations include those inherent in the use of convenience sampling, namely the potential of bias from underrepresentation or overrepresentation of particular groups within the sample, and lack of information on nonparticipants. The sample may not be representative of the population at large of breastfeeding women in the US, and was composed primarily of middle- to high-income Caucasian mothers. A strength was the use of a customized data-collection instrument for information on herbal and pharmaceutical galactagogues. This is the first research published to date on knowledge about and use of galactagogues in the US. The use of convenience sampling, though limited in generalizability, illustrates the utility of preliminary surveys to inform more detailed research protocols.

Exploring further the reasons that mothers may make use of particular galactagogues would contribute to health-education efforts, and additional experimental and observational research is required on the safety and efficacy of these. While there is a need for further evaluation of botanical galactagogues, particularly for those most widely used ${ }^{85}$ there are unfortunately barriers to conducting appropriate research, eg, the mismatch between clinical research protocols and traditional preparations used for study, as well as a lack of dedicated funding for this work. ${ }^{86}$

This study brings further awareness of alternative methods of lactation support that breastfeeding mothers avail themselves of in an effort to improve lactation outcomes and overcome perceived or physiological barriers to breastfeeding. Breast milk is superior to all artificial food in terms of nutritional value, and the World Health Organization, among other international bodies, strongly advises that in the first 6 months of life, a baby should be fed only with breast milk, ${ }^{87}$ yet women continue to experience barriers to doing so. Our findings emphasize that mothers, along with health professionals, such as pediatricians, nutritionists, obstetricians, nurses, and lactation consultants, need evidence-based information regarding herbal and pharmaceutical galactagogues, in order to make informed choices to improve lactation and successful breastfeeding.

\section{Author contributions}

All authors contributed toward data analysis, drafting and revising the paper and agree to be accountable for all aspects of the work.

\section{Disclosure}

The authors report no conflicts of interest in this work.

\section{References}

1. Bass PF 3rd. Evidence-based support for breastfeeding. 2015 Available from: http://contemporarypediatrics.modernmedicine.com/ contemporary-pediatrics/news/evidence-based-support-breastfeeding. Accessed January 24, 2017.

2. Dewey KG, Nommsen-Rivers LA, Heinig MJ, Cohen RJ. Risk factors for suboptimal infant breastfeeding behavior, delayed onset of lactation, and excess neonatal weight loss. Pediatrics. 2003;112(3 Pt 1):607-619.

3. Abrahams SW, Labbok MH. Breastfeeding and otitis media: a review of recent evidence. Curr Allergy Asthma Rep. 2011;11(6):508-512.

4. Horta BL, Victora CG. Long-Term Effects of Breastfeeding: A Systematic Review. Geneva: World Health Organization; 2013.

5. Horta BL, Victora CG. Short-Term Effects of Breastfeeding: A Systematic Review on the Benefits of Breastfeeding on Diarrhoea and Pneumonia Mortality. Geneva: World Health Organization; 2013.

6. Victora CG, Bahl R, Barros AJ, et al. Breastfeeding in the 21st century: epidemiology, mechanisms, and lifelong effect. Lancet. 2016; 387(10017):475-490.

7. Perrine CG, Galuska DA, Dohack JL, et al. Vital signs: improvements in maternity care policies and practices that support breastfeeding: United States, 2007/2013. MMWR Morb Mortal Wkly Rep. 2015;64(39): 1112-1117.

8. [No authors listed]. Breastfeeding and the use of human milk. Pediatrics. 2012;129(3):e827-e841. 
9. Centers for Disease Control and Prevention. Breastfeeding report cards. 2014. Available from: http://www.cdc.gov/breastfeeding/data/ reportcard.htm. Accessed January 24, 2017.

10. Jolly K, Ingram L, Khan KS, Deeks JJ, Freemantle N, MacArthur C. Systematic review of peer support for breastfeeding continuation: metaregression analysis of the effect of setting, intensity, and timing. BMJ. 2012;344:d8287.

11. Cross-Barnet C, Augustyn M, Gross S, Resnik A, Paige D. Long-term breastfeeding support: failing mothers in need. Matern Child Health J. 2012;16(9):1926-1932.

12. Busch DW, Logan K, Wilkinson A. Clinical practice breastfeeding recommendations for primary care: applying a tri-core breastfeeding conceptual model. J Pediatr Health Care. 2014;28(6):486-496.

13. Schaffir J, Czapla C. Survey of lactation instructors on folk traditions in breastfeeding. Breastfeed Med. 2012;7(4):230-233.

14. Skouteris H, Nagle C, Fowler M, Kent B, Sahota P, Morris H. Interventions designed to promote exclusive breastfeeding in high-income countries: a systematic review. Breastfeed Med. 2014;9(3):113-127.

15. Gatti L. Maternal perceptions of insufficient milk supply in breastfeeding. J Nurs Scholarsh. 2008;40(4):355-363.

16. Ahmed AH, Roumani AM, Szucs K, Zhang L, King D. The effect of interactive web-based monitoring on breastfeeding exclusivity, intensity, and duration in healthy, term infants after hospital discharge. J Obstet Gynecol Neonatal Nurs. 2016;45(2):143-154.

17. Lewallen LP, Dick MJ, Flowers J, et al. Breastfeeding support and early cessation. J Obstet Gynecol Neonatal Nurs. 2006;35(2):166-172.

18. Lou Z, Zeng G, Huang L, Wang Y, Zhou L, Kavanagh KF. Maternal reported indicators and causes of insufficient milk supply. J Hum Lact. 2014;30(4):466-473.

19. Pierro J, Abulaimoun B, Roth P, Blau J. Factors associated with supplemental formula feeding of breastfeeding infants during postpartum hospital stay. Breastfeed Med. 2016;11(4):196-202.

20. Tenfelde S, Zielinski R, Heidarisafa RL. Why WIC women stop breastfeeding? [sic] Analysis of maternal characteristics and time to cessation. Infant Child Adolesc Nutr. 2013;5(4):207-214.

21. Zapantis A, Steinberg JG, Schilit L. Use of herbals as galactagogues. J Pharm Pract. 2012;25(2):222-231.

22. Knoppert DC, Page A, Warren J, et al. The effect of two different domperidone doses on maternal milk production. J Hum Lact. 2013; 29(1):38-44.

23. Sim TF, Hattingh HL, Sherriff J, Tee LB. Perspectives and attitudes of breastfeeding women using herbal galactagogues during breastfeeding: a qualitative study. BMC Complement Altern Med. 2014;14:216.

24. Sim TF, Hattingh HL, Sherriff J, Tee LB. The use, perceived effectiveness and safety of herbal galactagogues during breastfeeding: a qualitative study. Int J Environ Res Public Health. 2015;12(9):11050-11071.

25. Sahin B, Kaymaz N, Yıldırım S. Herbal remedies for perceived inadequate milk supply are perhaps not as safe as women think: a brief case report. Women Birth. 2016;29(6):e133.

26. Zecca E, Zuppa AA, D'Antuono A, et al. Efficacy of a galactogogue containing silymarin-phosphatidylserine and galega in mothers of preterm infants: a randomized controlled trial. Eur J Clin Nutr. 2016; 70(10):1151-1154.

27. Haase B, Taylor SN, Mauldin J, Johnson TS, Wagner CL. Domperidone for treatment of low milk supply in breast pump-dependent mothers of hospitalized premature infants: a clinical protocol. J Hum Lact. 2016;32(2):373-381.

28. Sumanth M, Narasimharaju K. Evaluation of galactagogue activity of lactovedic: a polyherbal formulation. Int J Green Pharm. 2011;5(1): 61-64.

29. Michels KA, Mumford SL, Sundaram R, Bell EM, Bello SC, Yeung EH. Differences in infant feeding practices by mode of conception in a United States cohort. Fertil Steril. 2016;105(4):1014-1022.e1.

30. Derin DO, Ozel E. An investigation of galactagogues from the perspective of mothers who have a newborn child. Int J Health Sci Res. 2016; 6(5):268-278.

31. Anderson PO. The galactogogue bandwagon. J Hum Lact. 2013; 29(1):7-10.
32. Mortel M, Mehta SD. Systematic review of the efficacy of herbal galactogogues. J Hum Lact. 2013;29(2):154-162.

33. Grzeskowiak LE, Amir LH. Use of domperidone to increase breast milk supply further consideration of the benefit-risk ratio is required. J Hum Lact. 2015;31(2):315-316.

34. da Silva OP, Knoppert DC, Angelini MM, Forret PA. Effect of domperidone on milk production in mothers of premature newborns: a randomized, double-blind, placebo-controlled trial. CMAJ. 2001;164(1): $17-21$.

35. Bako IG, Mabrouk AM, Abubakar SM, Mohammed A. Lactogenic study of the ethyl-acetate fraction of Hibiscus sabdariffa Linn seed on pituitary prolactin level of lactating albino rats. Int J Appl Res Nat Prod. 2013;6(2):30-37.

36. Damanik R, Wahlqvist ML, Wattanapenpaiboon N. Lactagogue effects of torbangun, a Bataknese traditional cuisine. Asia Pac J Clin Nutr. 2006; 15(2):267-274.

37. Gabay MP. Galactogogues: medications that induce lactation. J Hum Lact. 2002;18(3):274-279.

38. Grzeskowiak LE, Dalton JA, Fielder AL. Factors associated with domperidone use as a galactogogue at an Australian tertiary teaching hospital. J Hum Lact. 2015;31(2):249-253.

39. Mannion C, Mansell D. Breastfeeding self-efficacy and the use of prescription medication: a pilot study. Obstet Gynecol Int. 2012;2012: 562704 .

40. Osadchy A, Moretti ME, Koren G. Effect of domperidone on insufficient lactation in puerperal women: a systematic review and metaanalysis of randomized controlled trials. Obstet Gynecol Int. 2012; 2012:642893.

41. Wan EW, Davey K, Page-Sharp M, Hartmann PE, Simmer K, Ilett KF. Dose-effect study of domperidone as a galactagogue in preterm mothers with insufficient milk supply, and its transfer into milk. Br J Clin Pharmacol. 2008;66(2):283-289.

42. Hale TW, Hartmann PE. Hale and Hartmann's Textbook of Human Lactation. Plano, TX: Hale Publishing; 2007.

43. Buddha S, Noffke B, Brown D Jr, Westerhoff M. Breast feeding, hypogalactia and molecular exploration of herbal galactogogues. Planta Med. 2012;78(11):CL60.

44. Forinash AB, Yancey AM, Barnes KN, Myles TD. The use of galactogogues in the breastfeeding mother. Ann Pharmacother. 2012;46(10): 1392-1404.

45. Sim TF, Sherriff J, Hattingh HL, Parsons R, Tee LB. The use of herbal medicines during breastfeeding: a population-based survey in Western Australia. BMC Complement Altern Med. 2013;13:317.

46. Budzynska K, Filippelli AC, Sadikova E, Low Dog T, Gardiner P. Use and factors associated with herbal/botanical and nonvitamin/nonmineral dietary supplements among women of reproductive age: an analysis of the Infant Feeding Practices study II. J Midwifery Womens Health. 2016;61(4):419-426.

47. Reeder C, LeGrand A, O'Connor-Vonn SK. The effect of fenugreek on milk production and prolactin levels in mothers of preterm infants. Clin Lact (Amarillo). 2013;4(4):159-165.

48. Turky1lmaz C, Onal E, Hirfanoglu IM, et al. The effect of galactagogue herbal tea on breast milk production and short-term catch-up of birth weight in the first week of life. J Altern Complement Med. 2011;17(2): 139-142.

49. Zuppa AA, Sindico P, Orchi C, Carducci C, Cardiello V, Romagnoli C. Safety and efficacy of galactogogues: substances that induce, maintain and increase breast milk production. J Pharm Pharm Sci. 2010; 13(2):162-174.

50. Axelsson PB, Bjerrum F, Løkkegaard EC. Treatment of insufficient lactation is often not evidence-based. Dan Med J. 2014;61(7):A4869.

51. Cameron SL, Taylor RW, Gray AR, Taylor BJ, Heath AL. Exclusive breastfeeding to six months: results from a randomised controlled trial including lactation consultant support. FASEB J. 2013; 27(1 Suppl):1b345.

52. Bonuck K, Stuebe A, Barnett J, Labbok MH, Fletcher J, Bernstein PS. Effect of primary care intervention on breastfeeding duration and intensity. Am J Public Health. 2014;104 (Suppl 1):S119-S127. 
53. Amir LH. Breastfeeding: managing 'supply' difficulties. Aust Fam Physician. 2006;35(9):686-689.

54. Flaherman VJ, Beiler JS, Cabana MD, Paul IM. Relationship of newborn weight loss to milk supply concern and anxiety: the impact on breastfeeding duration. Matern Child Nutr. 2016;12(3):463-472.

55. Riddle SW, Nommsen-Rivers LA. A case control study of diabetes during pregnancy and low milk supply. Breastfeed Med. 2016;11(2):80-85.

56. Froh E, Dahlmeier K, Spatz DL. NICU nurses and lactation-based support and care. Adv Neonatal Care. Epub 2016 Dec 27.

57. Dennison BA, Nguyen TQ, Gregg DJ, Fan W, Xu C. The impact of hospital resources and availability of professional lactation support on maternity care: results of breastfeeding surveys 2009-2014. Breastfeed Med. 2016;11(9):479-486.

58. Busch DW, Logan K, Wilkinson A. Clinical practice breastfeeding recommendations for primary care: applying a tri-core breastfeeding conceptual model. J Pediatr Health Care. 2014;28(6):486-496.

59. Patnode CD, Henninger ML, Senger CA, Perdue LA, Whitlock EP. Primary Care Interventions to Support Breastfeeding: Updated Systematic Review for the U.S. Preventive Services Task Force. Rockville (MD): Agency for Healthcare Research and Quality; 2016.

60. Asztalos EV, Campbell-Yeo M, daSilva OP, Kiss A, Knoppert DC, Ito $\mathrm{S}$. Enhancing breast milk production with domperidone in mothers of preterm neonates (EMPOWER trial). BMC Pregnancy Childbirth. 2012;12:87.

61. Budzynska K, Gardner ZE, Dugoua JJ, Low Dog T, Gardiner P. Systematic review of breastfeeding and herbs. Breastfeed Med. 2012;7(6):489-503.

62. Ingram J, Taylor H, Churchill C, Pike A, Greenwood R. Metoclopramide or domperidone for increasing maternal breast milk output: a randomised controlled trial. Arch Dis Child Fetal Neonatal Ed. 2012; 97(4):F241-F245.

63. Wagner CL, Murphy PK, Haase B, Barreira J, Taylor SN. Domperidone in the treatment of low milk supply in mothers of critically ill neonates. Breastfeed Med. 2011;6 (Suppl 1):S21.

64. American College of Obstetricians and Gynecologists. Committee opinion no 570 - breastfeeding in underserved women: increasing initiation and continuation of breastfeeding. Obstet Gynecol. 2013;122(2 Pt 1): 423-427.

65. Prior E, Santhakumaran S, Gale C, Philipps LH, Modi N, Hyde MJ. Breastfeeding after cesarean delivery: a systematic review and metaanalysis of world literature. Am J Clin Nutr. 2012;95(5):1113-1135.

66. US Department of Health and Human Services. Women's Health US A 2013. Rockville (MD): DHHS; 2013.

67. Babendure JB, Reifsnider E, Mendias E, Moramarco MW, Davila YR. Reduced breastfeeding rates among obese mothers: a review of contributing factors, clinical considerations and future directions. Int Breastfeed J. 2015;10:21.

68. Smolina K, Morgan SG, Hanley GE, Oberlander TF, Mintzes B. Postpartum domperidone use in British Columbia: a retrospective cohort study. CMAJ Open. 2016;4(1):E13-E19.
69. Bazzano AN, Hofer R, Thibeau S, Gillispie V, Jacobs M, Theall KP A review of herbal and pharmaceutical galactagogues for breast-feeding. Ochsner J. 2016;16(4):511-524.

70. Posadzki P, Watson LK, Ernst E. Adverse effects of herbal medicines: an overview of systematic reviews. Clin Med (Lond). 2013;13(1):7-12.

71. Chambers JA, McInnes RJ, Hoddinott P, Alder EM. A systematic review of measures assessing mothers' knowledge, attitudes, confidence and satisfaction towards breastfeeding. Breastfeed Rev. 2007;15(3):17-25.

72. Kornides M, Kitsantas P. Evaluation of breastfeeding promotion, support, and knowledge of benefits on breastfeeding outcomes. J Child Health Care. 2013;17(3):264-273.

73. Romano AM. A changing landscape: implications of pregnant women's Internet use for childbirth educators. J Perinat Educ. 2007; 16(4):18-24.

74. Chapman DJ, Pérez-Escamilla R. Identification of risk factors for delayed onset of lactation. J Am Diet Assoc. 1999;99(4):450-456.

75. Westfall R. Galactagogue herbs: a qualitative study and review. Can $J$ Midwifery Res Pract. 2003;2(2):22-27.

76. Staikou C, Stamelos M, Stavroulakis E. Impact of anaesthetic drugs and adjuvants on ECG markers of torsadogenicity. Br J Anaesth. 2014; 112(2):217-230.

77. Centers for Disease Control and Prevention. Breastfeeding report cards. 2013. Available from: http://www.cdc.gov/breastfeeding/data/ reportcard.htm. Accessed January 24, 2017.

78. Royal Pharmaceutical Society. A new mother is having trouble producing breast milk. What options are available to help? Clin Pharm. 2010;2(7-8):260.

79. Tabares FP, Bedoya Jaramillo JB, Ruiz-Cortés ZT. Pharmacological overview of galactogogues. Vet Med Int. 2014;2014:602894.

80. Bishop J. Is domperidone safe for breastfeeding mothers? J Midwifery Womens Health. 2004:49(5):461.

81. Campbell-Yeo ML, Allen AC, Joseph KS, Ledwidge JM, Allen VM, Dooley KC. Study protocol: a double blind placebo controlled trial examining the effect of domperidone on the composition of breast milk [NCT00308334]. BMC Pregnancy Childbirth. 2006;6:17.

82. Choi JS, Han JY, Ahn HK, et al. Fetal and neonatal outcomes in women taking domperidone during pregnancy. J Obstet Gynaecol. 2013;33(2):160-162.

83. Henderson A. Domperidone: discovering new choices for lactating mothers. AWHONN Lifelines. 2003;7(1):54-60.

84. Anderson PO, Valdes V. A critical review of pharmaceutical galactagogues. Breastfeed Med. 2007;2(4):229-242.

85. Abascal K, Yarnell E. Botanical galactagogues. Altern Complement Ther. 2008;14(6):288-294.

86. Abascal K, Yarnell E. Dilemmas of traditional botanical research. HerbalGram. 2002;55:46-54.

87. Chung M, Raman G, Trikalinos T, Lau J, Ip S. Interventions in primary care to promote breastfeeding: an evidence review for the U.S. Preventive Services Task Force. Ann Intern Med. 2008;149(8):565-582.
International Journal of Women's Health

\section{Publish your work in this journal}

The International Journal of Women's Health is an international, peerreviewed open-access journal publishing original research, reports, editorials, reviews and commentaries on all aspects of women's healthcare including gynecology, obstetrics, and breast cancer. The manuscript management system is completely online and includes

\section{Dovepress}

a very quick and fair peer-review system, which is all easy to use. Visit http://www.dovepress.com/testimonials.php to read real quotes from published authors. 